\title{
Volumes with Piecewise Quadratic Medial Surface Transforms: Computation of Boundaries and Trimmed Offsets
}

\author{
Bohumír Bastl $^{\mathrm{a}}$, Bert Jüttler ${ }^{\mathrm{b}, 1}$, Jiří Kosinka ${ }^{\mathrm{c}}$ and Miroslav Lávička ${ }^{\mathrm{a}}$ \\ a University of West Bohemia, Faculty of Applied Sciences, Department of Mathematics, \\ Univerzitní 8, 30100 Plzeň, Czech Republic \\ b Johannes Kepler University, Institute of Applied Geometry, \\ Altenberger Str. 69, 4040 Linz, Austria \\ c University of Oslo, Centre of Mathematics for Applications, \\ P.O. Box 1053 Blindern, NO-0316 Oslo, Norway
}

\begin{abstract}
MOS surfaces are rational surfaces in $\mathbb{R}^{3,1}$ which provide rational envelopes of the associated two-parameter family of spheres. Moreover, all the offsets admit rational parameterizations as well. Recently, it has been proved that quadratic triangular Bézier patches in $\mathbb{R}^{3,1}$ are MOS surfaces. Following this result, we describe an algorithm for computing an exact rational envelope of a two-parameter family of spheres given by a quadratic patch in $\mathbb{R}^{3,1}$. The main focus of this paper is given to geometric aspects of the algorithm. Since these patches are capable of producing $C^{1}$ smooth approximations of medial surface transforms of spatial domains, we use this algorithm to generate rational approximations of envelopes of general medial surface transforms. One of the main advantages of this approach to offsetting is the fact that the trimming procedure becomes considerably simpler.
\end{abstract}

Keywords: Quadratic Bézier triangles, MOS surfaces, trimmed offsets

\section{Introduction}

Generating valid tool paths in NURBS form has become a universal standard in technical applications such as CNC machining in recent years. Hence, shape (curve, surface or volume) offsets and corresponding algorithms have been widely studied in Computer-Aided Design and Manufacturing, see [1, 2, 3, 4, 5, 6, $7,8]$ and references therein for more details. However, free-form NURBS shapes do not possess rational offsets in general and thus suitable approximation techniques, often based on rational curves or surfaces of relatively low degree, are used - see e.g. $[9,10,11,12,13,14]$. Nevertheless, these offset approximation techniques suffer from several shortcomings. First, one needs to approximate each offset independently. Moreover, the constructed approximation of offsets to the same base shape may not have a constant distance to each other, which is not acceptable in some applications, e.g. architecture. Second, although a rational approximation of an offset is computed, the so called trimming, i.e., the detection and elimination of self-intersections, is still a

\footnotetext{
${ }^{1}$ Corresponding author. E-mail: bert.juettler@jku.at, phone/fax: +43732 24689178 / 29162, Homepage: www.ag.jku.at.
}

very difficult problem. Let us emphasize that the approximation can possess self-intersections even though the original offset has none. Even in the case of curves this leads to challenging computational and time-consuming problems and the situation is even more complicated for surfaces, $\mathrm{cf}$. $[15,16,17]$. The purpose of this paper is to identify an approach that avoids these problems.

The approximate techniques for offsets are now widely used in CAD systems since they are capable of dealing with problems appearing in technical practice mentioned above - but usually at the expense of great computational effort. Therefore, it is worthwhile to investigate exact techniques as well, i.e., to study shapes with exact rational offsets. These can be subsequently used for formulating suitable approximation techniques for freeform shapes. Compared to classical approximation techniques, not offsets but the base shape is approximated and it is guaranteed that all corresponding offsets are rational. Therefore, only one approximation step is required even if more than one offset is needed. Moreover, all the offsets are at a constant distance from each other.

Shapes with rational offsets have been studied for many years. In the case of planar curves, the class of Pythagorean Hodograph $(\mathrm{PH})$ curves as polynomial curves possessing rational offset curves and polynomial arc-length functions was introduced in [18]. A thorough analysis of PH curves has followed - see e.g. $[19,20,21,12,22]$. Later, the concept of polynomial planar PH curves was generalized to space PH curves $([23,24,25,26])$ and to rational PH curves $([27,28,29])$. Analogously, the notion of rational surfaces with rational offsets, the so called Pythagorean Normal vector (PN) surfaces, was introduced in [27]. More details about PN surfaces can be found in [30, 31, 32]. For a survey of shapes with Pythagorean normals property (i.e., possessing rational offsets) see [33].

Later, it has been proved in [34] that surfaces with Linear field of Normal vectors (LN surfaces), introduced in [35], provide rational convolution surfaces with an arbitrary rational surface. Since spheres admit rational descriptions, LN surfaces possess exact rational offsets. This result was applied in [36] to constructing exact rational offsets of LN surfaces. As observed recently $([37,38])$, all non-developable polynomial quadratic sur- 
faces belong to the class of LN surfaces and therefore possess rational offsets. Motivated by the fact that approximation by quadratic Bézier surfaces provides a good compromise between curvature reproduction and computational cost and offers an extra feature, i.e., the PN property, these patches were used in [39] for formulating an algorithm for generating rational approximations to offsets of general free-form surfaces.

Even though PH curves and PN surfaces admit rational offsets, the usually most costly ingredient, the trimming, still needs to be performed as in the case of approximation techniques. An alternative approach to the problem in the curve case based on the medial axis transform (MAT) of a planar domain, introduced in [40], was formulated in [41] and [42]. There is a one-to-one correspondence between the MAT in three-dimensional Minkowski space $\mathbb{R}^{2,1}$ and the object boundary, i.e., for a given geometric object there is a unique MAT and conversely, the object can be directly reconstructed from its MAT. The main advantages of this description follow from the dimensional reduction while topological properties are maintained. As observed in [42, 41], if a segment of the medial axis transform is an MPH (Minkowski Pythagorean Hodograph) curve, then the associated branches of the domain boundaries are segments of rational curves. In addition, all offsets of the domain boundaries have also this property. A thorough analysis of MPH curves has followed in [43, 44, 45, 46, 47, 48]. Moreover, using the MAT representation makes the trimming procedure for the inner offsets very simple - only those parts of the MAT where the corresponding circle radius is less than the offset distance $\delta$ have to be trimmed. For more details see e.g. [49, 50, 51, 52, 53, 54, 55].

The situation in three-dimensional space has become an active research area recently, since the so called MOS surfaces as a spatial analogy of MPH curves were introduced in [56]. The medial surface transform (MST) of a volume is the set of surface patches (or curve segments) in four-dimensional Minkowski space $\mathbb{R}^{3,1}$ such that each point of these surfaces/curves represents the center and the radius of a maximal ball inscribed into the domain. The MST covers the structure of the domain and thus can be used in all sorts of geometric modelers (constructive solid geometry (CSG) and boundary representation (B-rep)) as a useful tool. The distinguishing property of MOS surfaces is that if considered as the MST of a volume, the associated envelope and its offsets admit exact rational parameterization. Later, it was proved in [57] that quadratic triangular Bézier surfaces in $\mathbb{R}^{3,1}$ possess the MOS property and a related study followed in [58, 59].

Following the results of [57], [39] and [60], we describe an efficient algorithm for computing boundaries and trimmed offsets of volumes with piecewise quadratic medial surface transforms. Considering general (free-form) medial surface transforms in $\mathbb{R}^{3,1}$, we produce their $C^{1}$ approximations using quadratic Bézier patches (cf. [61, 62]), which gives us an extra feature - the MOS property. Thus we have a guarantee on the rationality of the volume boundaries as well as all offsets. The presented technique also simplifies the computation of self-intersections of inner offsets and the follow-up trimming procedure considerably.

As far as we are aware of the literature, this paper is the first paper which proposes a working method for offset trimming which is based on the medial surface transform. Clearly, this is a very natural approach to address this problem. Still, the existing literature mostly does this in a different way, by first offsetting the surfaces and then eliminating the self-intersecting parts. We believe that the medial surface-based approach is far more elegant.

Modeling volumes directly by their MST (or even MOS patches) might become an interesting and also a practical volume designing tool. However, it is not straightforward for an inexperienced user to design volumes this way. Even though the bisector surface is quite easy to control, we emphasize that the MST is an object lying in 4-space.

The remainder of this paper is organized as follows. Section 2 recalls some basic facts concerning medial surface transforms, envelopes of two-parameter families of spheres and MOS surfaces. Section 3 is devoted to quadratic triangular Bézier surfaces in $\mathbb{R}^{3,1}$, their (isotropic) normals and isotropic Gauss images. In this section, we also formulate and discuss an algorithm for envelope computation for quadratic MSTs. The algorithm is then demonstrated on several examples in Section 4. Finally, we conclude the paper.

\section{Preliminaries}

We recall the relation between the four-dimensional Minkowski space $\mathbb{R}^{3,1}$ and the medial surface transform (MST) of a volume. We then consider rational patches on sheets of the MST and the computation of the corresponding patches on the boundary surface of the volume.

\subsection{Medial surface transform}

The four-dimensional Minkowski space $\mathbb{R}^{3,1}$ is the fourdimensional real affine space which is equipped with the indefinite inner product

$$
\langle\mathbf{u}, \mathbf{v}\rangle=\mathbf{u}^{\top} \mathbf{J} \mathbf{v}=u_{1} v_{1}+u_{2} v_{2}+u_{3} v_{3}-u_{4} v_{4}
$$

where $\mathbf{J}=\operatorname{diag}(1,1,1,-1)$. The hyperplane $x_{4}=0$ is a threedimensional subspace, and the restriction of the inner product to this hyperplane is the usual Euclidean inner product. We identify this hyperplane with the three-dimensional Euclidean space. More precisely, any point $\mathbf{x}=\left(x_{1}, x_{2}, x_{3}\right)^{\top} \in \mathbb{R}^{3}$ is equivalently considered as a point $\mathbf{x}=\left(x_{1}, x_{2}, x_{3}, 0\right)^{\top} \in \mathbb{R}^{3,1}$.

For any point $\mathbf{p}=\left(p_{1}, p_{2}, p_{3}, p_{4}\right)^{\top} \in \mathbb{R}^{3,1}$, let $\mathbf{p}^{\prime}=$ $\left(p_{1}, p_{2}, p_{3}\right)^{\top}$ be its projection into $\mathbb{R}^{3}$. The set

$$
\mathcal{B}_{\mathbf{p}}=\left\{\mathbf{x} \in \mathbb{R}^{3}:\langle\mathbf{p}-\mathbf{x}, \mathbf{p}-\mathbf{x}\rangle \leq 0\right\}
$$

is the oriented ball with center $\mathbf{p}^{\prime}$ and radius $\left|p_{4}\right|$. The sign of the radius $p_{4}$ defines the orientation of the ball. Its boundary is the oriented sphere $\mathcal{S}_{\mathbf{p}}=\delta \mathcal{B}_{\mathbf{p}}$ which contains all points $\mathbf{x} \in \mathbb{R}^{3}$ satisfying

$$
\langle\mathbf{p}-\mathbf{x}, \mathbf{p}-\mathbf{x}\rangle=0 .
$$

Any oriented ball in $\mathbb{R}^{3}$ can be identified with a unique point in the four-dimensional Minkowski space $\mathbb{R}^{3,1}$.

We consider a bounded volume $V$ with a smooth boundary $\delta V$. The set of all balls with non-negative radius (i.e., with nonnegative orientation) which are contained in $V$ is partially ordered with respect to inclusion. The maximal elements of this set 


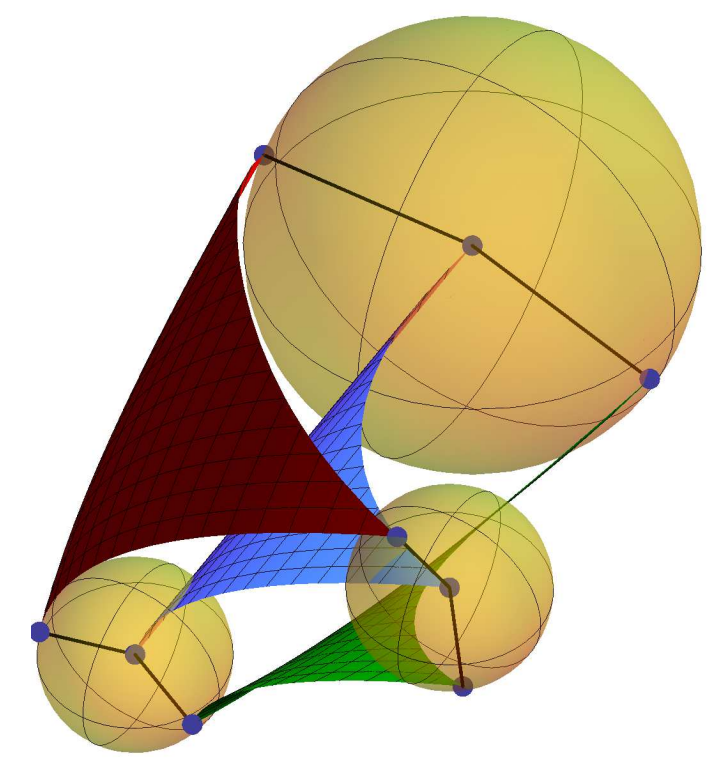

Figure 1: The bisector surface (blue) and two branches (red and green) of the envelope surface forming a boundary of a volume $V$.

form the medial surface transform (MST) of $V$, cf. Fig. 1. We consider them as points in Minkowski space, which then form the set $\operatorname{MST}(V) \subset \mathbb{R}^{3,1}$.

The medial surface transform of the volume $V$ consists of components of dimensions two, one, and zero, which are called sheets, seams and junctions, respectively. Their points correspond to maximal inscribed balls which - in the generic case touch the boundary in two, three, and four points, respectively. The sheets meet in seams, and the seams meet in junctions.

In the remainder of this paper we consider a two-dimensional rational surface patch

$$
\mathbf{p}: \Omega \rightarrow \mathbb{R}^{3,1}:(u, v) \mapsto \mathbf{p}(u, v)
$$

with domain $\Omega \subseteq \mathbb{R}^{2}$ and parameters $u, v$, which defines a rational parameterization of a subset of the interior of a sheet. Every ball $\mathcal{B}_{\mathbf{p}(u, v)}$ touches the boundary $\delta V$ of the volume in two points. The contact points form two surface patches on the boundary. The centers $\mathbf{p}^{\prime}(u, v)$ of the balls form the bisector surface of these two surface patches - see Fig. 1.

\subsection{Envelopes of two-parameter families of spheres}

Given a patch (4) of the medial surface transform, we are interested in computing the associated patches of the boundary surface, see Fig. 1. These two patches are obtained as the envelope of the two-parameter family of spheres

$$
\left\{\mathcal{S}_{\mathbf{p}(u, v)}:(u, v) \in \Omega\right\} .
$$

By differentiating equation (3) of the spheres we obtain two equations

$$
\left\langle\partial_{u} \mathbf{p}, \mathbf{p}-\mathbf{x}\right\rangle=0, \quad\left\langle\partial_{v} \mathbf{p}, \mathbf{p}-\mathbf{x}\right\rangle=0
$$

which - along with (3) - characterize the points $\mathbf{x}$ of the envelope surfaces. Recall that $\mathbf{x} \in \mathbb{R}^{3}$ is a point in the hyperplane $x_{4}=0$. The substitution

$$
\mathbf{x}=\mathbf{p}-\frac{p_{4}}{n_{4}} \mathbf{n}
$$

transforms the three equations (3) and (6) into a homogeneous non-linear system of equations

$$
\begin{aligned}
\langle\mathbf{n}, \mathbf{n}\rangle & =0 \quad \text { and } \\
\left\langle\mathbf{n}, \partial_{u} \mathbf{p}\right\rangle & =0, \quad\left\langle\mathbf{n}, \partial_{v} \mathbf{p}\right\rangle=0
\end{aligned}
$$

for the vector $\mathbf{n} \in \mathbb{R}^{3,1}$.

The two equations (9) express the fact that the vector $\mathbf{n}$ is orthogonal to the two-dimensional tangent plane of the surface patch $\mathbf{p}$ with respect to the Minkowski inner product (1). The first equation (8) restricts the solutions to isotropic vectors (vectors of zero length with respect to the Minkowski metric). Consequently, the non-trivial solutions of the system are the isotropic normal vectors of the surface.

Summing up, the points $\mathbf{x}$ of the envelope surfaces can be generated by

1. finding all non-trivial isotropic normal vectors and

2. computing $\mathbf{x}$ from (7).

Note that any nontrivial solution of (8) satisfies $n_{4} \neq 0$.

\subsection{MOS surfaces}

The sub-determinants ${ }^{2}$

$$
P_{i j}=\operatorname{det}\left(\begin{array}{cc}
\partial_{u} p_{i} & \partial_{v} p_{i} \\
\partial_{u} p_{j} & \partial_{v} p_{j}
\end{array}\right), \quad i, j=1, \ldots, 4,
$$

of the $4 \times 2$ matrix $\left(\partial_{u} \mathbf{p}, \partial_{v} \mathbf{p}\right)$ formed by partial derivative vectors can be used to express the solutions of the system (8)-(9),

$$
\mathbf{n}_{ \pm}(\mu)=\mu\left(\begin{array}{c}
P_{12} P_{24}+P_{13} P_{34} \pm \sqrt{C} P_{23} \\
P_{21} P_{14}+P_{23} P_{34} \pm \sqrt{C} P_{31} \\
P_{31} P_{14}+P_{32} P_{24} \pm \sqrt{C} P_{12} \\
-\left(P_{23}^{2}+P_{31}^{2}+P_{12}^{2}\right)
\end{array}\right), \quad \mu \in \mathbb{R}
$$

with

$$
C=P_{12}^{2}+P_{31}^{2}+P_{23}^{2}-P_{14}^{2}-P_{24}^{2}-P_{34}^{2} .
$$

The number of linearly independent solutions depends on the sign of $C$ as follows.

- If $C>0$, then we obtain two families of solutions. The orthogonal complement space of the two-dimensional tangent plane, which is described by (9), intersects the quadratic cone of isotropic vectors (8) in two lines. We obtain two points of the envelope surfaces. The tangent plane is said to be space-like.

- If $C=0$, then we obtain one family of solutions. The orthogonal complement space of the two-dimensional tangent plane intersects the quadratic cone of isotropic vectors (8) in one line. We obtain one point of the envelope surfaces. The tangent plane is said to be light-like.

\footnotetext{
${ }^{2}$ These sub-determinants are the Plücker coordinate of the line (cf. [63]) in the plane at infinity, which is spanned by the two derivative vectors.
} 
- If $C<0$, then we obtain no non-trivial solutions. The orthogonal complement space of the two-dimensional tangent plane intersects the quadratic cone of isotropic vectors only in the origin, which is the apex of the cone. The tangent plane is said to be time-like.

If the surface patch (4) describes a subset of the interior of a MST sheet, then $C(u, v)>0$ is satisfied for all $(u, v) \in \Omega$. All points in the domain correspond to space-like tangent planes.

Consequently, for all $(u, v) \in \Omega$, two solutions (11) with two associated points of the envelope surface exist. Moreover, the bisector surface is regular since

$$
\left\|\partial_{u} \mathbf{p}^{\prime} \times \partial_{v} \mathbf{p}^{\prime}\right\|^{2}=P_{23}^{2}+P_{31}^{2}+P_{12}^{2} \geq C .
$$

Finally we recall a definition from [56] which generalizes the notion of Minkowski Pythagorean hodograph (MPH) curves. If there exists a polynomial $\sigma(u, v)$ such that $C=\sigma^{2}$, then the parameterization of the envelope surfaces, which is obtained by combining (11) with (7), is rational. The given surface $\mathbf{p}$ is then a MOS surface, i.e., a Medial surface Obeying the Sum of squares condition

$$
C=\sigma^{2} \Longleftrightarrow P_{12}^{2}+P_{31}^{2}+P_{23}^{2}=\sigma^{2}+P_{14}^{2}+P_{24}^{2}+P_{34}^{2} .
$$

\section{Quadratic patches as medial surface transforms}

A quadratic patch in $\mathbb{R}^{3,1}$ is defined by the power basis representation

$$
\mathbf{p}(u, v)=\frac{1}{2} \mathbf{q}_{20} u^{2}+\mathbf{q}_{11} u v+\frac{1}{2} \mathbf{q}_{02} v^{2}+\mathbf{q}_{10} u+\mathbf{q}_{01} v+\mathbf{q}_{00},
$$

with the coefficient vectors $\mathbf{q}_{i j} \in \mathbb{R}^{4}$, where the parameters $u, v$ span a suitable domain. We will mostly work with the standard triangular domain $\triangle \subset \mathbb{R}^{2}$ given by $u \in[0,1]$ and $v \in[0,1-$ $u$. According to the affine classification of quadratic patches [64], there exist 42 different types of quadratic patches. In the remainder of this paper we exclude developable patches (planes, cones and parabolic cylinders) from our considerations.

\subsection{Normals}

The two equations (9) take the form

$$
\begin{aligned}
& f_{\mathbf{n}}(u, v)=\left\langle\mathbf{n}, \mathbf{q}_{20}\right\rangle u+\left\langle\mathbf{n}, \mathbf{q}_{11}\right\rangle v+\left\langle\mathbf{n}, \mathbf{q}_{10}\right\rangle=0 \\
& g_{\mathbf{n}}(u, v)=\left\langle\mathbf{n}, \mathbf{q}_{11}\right\rangle u+\left\langle\mathbf{n}, \mathbf{q}_{02}\right\rangle v+\left\langle\mathbf{n}, \mathbf{q}_{01}\right\rangle=0 .
\end{aligned}
$$

For any given normal $\mathbf{n}$, they form a linear system for the parameters $(u, v)$.

The number of solutions $(u, v)$ depends on the normal $\mathbf{n}$. Since the system is homogeneous with respect to $\mathbf{n}$, we consider the normals as points in a three-dimensional real projective space

$$
\mathcal{N}=P^{3}(\mathbb{R})
$$

This three-dimensional space is the hyperplane at infinity of the four-dimensional Minkowski space.
Let

$$
d(\mathbf{n})=\mathbf{n}^{\top} \mathbf{J}\left(\mathbf{q}_{20} \mathbf{q}_{02}^{\top}-\mathbf{q}_{11} \mathbf{q}_{11}^{\top}\right) \mathbf{J} \mathbf{n}
$$

be the determinant of the linear system (16). Further, let

$$
\begin{aligned}
& d_{u}(\mathbf{n})=\mathbf{n}^{\top} \mathbf{J}\left(\mathbf{q}_{11} \mathbf{q}_{01}^{\top}-\mathbf{q}_{02} \mathbf{q}_{10}^{\top}\right) \mathbf{J n} \\
& d_{v}(\mathbf{n})=\mathbf{n}^{\top} \mathbf{J}\left(\mathbf{q}_{10} \mathbf{q}_{11}^{\top}-\mathbf{q}_{01} \mathbf{q}_{20}^{\top}\right) \mathbf{J n}
\end{aligned}
$$

be the two determinants generated by applying Cramer's rule.

First we describe the relation between the normals $\mathbf{n}$ and the number of solutions of the system (16).

- The set

$$
\mathcal{S}=\{\mathbf{n}: d(\mathbf{n})=0\}
$$

is called the set $\mathcal{S}$ of singular normals. It is a singular quadric surface in $\mathcal{N}$. $\mathcal{S}$ consists of all normals with no or non-unique solutions of (16). Consequently, if $\mathbf{n} \in \mathcal{N} \backslash \mathcal{S}$, then the set of solutions consists of a single point in the $u v-$ plane.

- The set

$$
\mathcal{E}=\left\{\mathbf{n}: d(\mathbf{n})=d_{u}(\mathbf{n})=d_{v}(\mathbf{n})=0\right\},
$$

which is contained in $\mathcal{S}$, is called the set of exceptional normals. It is a (possibly degenerate) cubic space curve, which is defined as the intersection of three quadric surfaces. $\mathcal{E}$ consists of all normals for which several solutions of (16) exist. If $\mathbf{n} \in \mathcal{E}$, then the set of all solutions forms a line in the $u v$-plane.

\subsection{The fibration of $\mathcal{N}$}

Next we study the sets

$$
\mathcal{F}_{(u, v)}=\left\{\mathbf{n} \in \mathcal{N}: f_{\mathbf{n}}(u, v)=g_{\mathbf{n}}(u, v)=0\right\}
$$

of normals which satisfy the two equations (16) for given values of $(u, v)$. They will be called the fibers. They form a partition of $\mathcal{N} \backslash \mathcal{S}$ into mutually disjoint subsets.

- If the quadratic surface is regular at $(u, v)$, then the fiber $\mathcal{F}_{(u, v)}$ is a line in $\mathcal{N}$. Indeed, it is defined as the intersection of the two linearly independent planes (9). At singular points, the fiber is a plane (if $\operatorname{rank}\left(\partial_{u} \mathbf{p}, \partial_{v} \mathbf{p}\right)=1$ ) or even the entire space $\mathcal{N}$ (if both derivatives vanish). The latter case occurs only for conical quadratic surfaces, which were excluded.

- Any line which is contained in a fiber $\mathcal{F}_{(u, v)}$ intersects the set $\mathcal{E}$ of exceptional normals in two (possibly conjugatecomplex) points (counted with multiplicities). Indeed, the two intersections of the line with the singular set $\mathcal{S}$ satisfy the equations for the given values $(u, v)$, hence these intersections belong to the exceptional set, too.

Example 1 We discuss several examples which are taken from the affine classification of quadratic patches in [64]. 

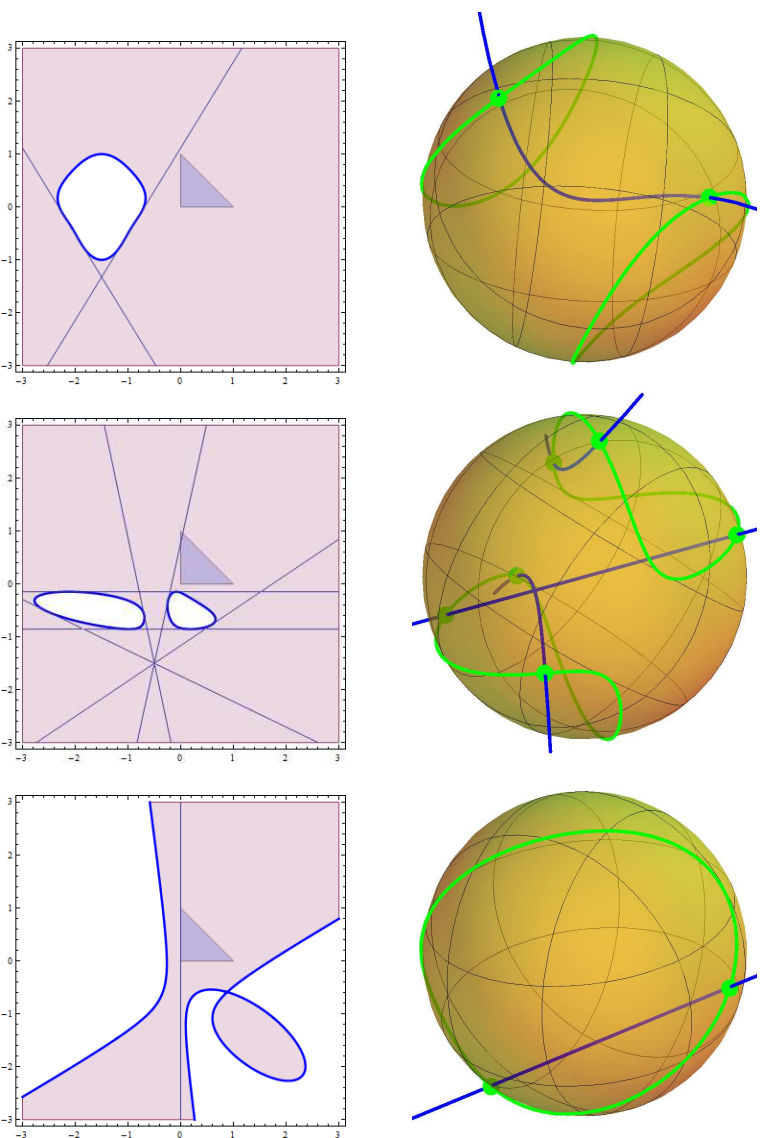

Figure 2: Left: Exceptional lines (thin blue), the curve $C(u, v)=0$ (thick blue) and the domain triangle $\triangle$. Right: The curve $\mathcal{S}_{\Sigma}$ (green), exceptional isotropic normals (green points) and the exceptional set $\mathcal{E}$ (blue). Top row: Example 1, patch $\mathbf{p}_{1}(u, v)$; Middle row: Example 1, patch $\mathbf{p}_{2}(u, v)$; Bottom row: Example 1, patch $\mathbf{p}_{3}(u, v)$.

1. Let us consider a non-hyperplanar patch given by

$$
\mathbf{p}_{1}(u, v)=\left(\left(u+\frac{3}{2}\right)^{2}+v, v^{2},\left(u+\frac{3}{2}\right) v, u+\frac{3}{2}\right)^{\top} .
$$

The exceptional set of this patch is a cubic space curve (see Fig. 2, top row).

2. Let us consider another non-hyperplanar patch

$$
\begin{aligned}
\mathbf{p}_{2}(u, v)= & \left(-u^{2}+u\left(v+\frac{1}{2}\right)+v^{2}+v+\frac{1}{4},\right. \\
& u^{2}+u+v^{2}+2 v+\frac{3}{4} \\
& \left.u^{2}+u-v^{2}+\frac{1}{4},-v-\frac{1}{2}\right)^{\top} .
\end{aligned}
$$

The exceptional set is formed by a conic and a line in this case (see Fig. 2, middle row).

3. Let us consider a hyperplanar patch

$$
\begin{aligned}
& \mathbf{p}_{3}(u, v)= \\
& \frac{1}{400}\left(289 u^{2}+17 u(17 v+40)+289 v^{2}+85 v+75,\right. \\
& -289 u^{2}-289 u v+289 v^{2}+595 v+325, \\
& 289 u^{2}+17 u(17 v+20)-5(51 v+25), \\
& (17 u-15)(17 u-17 v-5))^{\top}
\end{aligned}
$$

The exceptional set consists of three lines (see Fig. 2, bottom row).

\subsection{Isotropic normals}

So far we studied solutions of the system of equations (9) for a quadratic patch (15). Now we will analyze the consequences of the additional equation (8) for isotropic normals.

The set of isotropic normals (i.e., the set of normals satisfying (8)) forms an oval quadric $\Sigma$ in the three-dimensional projective space $\mathcal{N}$. Without loss of generality we may assume that the isotropic normals satisfy $n_{4}=1$. The oval quadric of isotropic normals can then be identified with the unit sphere

$$
0=\langle\mathbf{n}, \mathbf{n}\rangle=n_{1}^{2}+n_{2}^{2}+n_{3}^{2}-1 .
$$

We analyze the relation between the isotropic normals and the number of solutions of the system (8)-(9).

- The intersection curve of the set $\mathcal{S}$ and the isotropic quadric $\Sigma$ is the spherical quartic curve $\mathcal{S}_{\Sigma}=\mathcal{S} \cap \Sigma$ of isotropic singular normals, cf. Fig. 3 (right). It consists of all isotropic normals with no or non-unique solutions of (9). Consequently, if $\mathbf{n} \in \Sigma \backslash \mathcal{S}_{\Sigma}$, then the set of solutions consists of a single point in the $u v$-plane.

- The intersection points of the sphere of isotropic normals with the exceptional curve $\mathcal{E}, \mathcal{E}_{\Sigma}=\mathcal{E} \cap \Sigma$ are the - at most 6 - exceptional isotropic normals, Fig. 3 (right). If $\mathbf{n} \in \mathcal{E}$, then the set of all solutions forms a line in the $u v$-plane. We obtain at most 6 lines in the $u v$-plane, which will be called the exceptional lines in the parameter domain - see Fig. 3 (left).

- Consider a fiber $\mathcal{F}_{(u, v)}$ at a regular point $\mathbf{p}(u, v)$. If $C(u, v)$ is positive, zero, or negative, then the fiber intersect the unit sphere in two, one, or no points. The curve defined by $C(u, v)=0$ in the parameter domain thus corresponds to a spherical curve $\mathcal{L}_{\Sigma}$ where the fibers touch $\Sigma$. This is the curve of isotropic normals at points with light-like tangent planes - see Fig. 3 (right).

\subsection{The isotropic Gauss images}

Let us assume that the domain $\Omega$ of the surface patch contains only points satisfying $C(u, v)>0$. Consequently, all tangent planes are space-like, and all points are regular. This assumption is satisfied for surfaces which represent a patch of the interior of a sheet of the MST.

For each point $(u, v)$ we obtain two isotropic normals satisfying $n_{4}=1$ by choosing $\mu=-1 /\left(P_{23}^{2}+P_{31}^{2}+P_{12}^{2}\right)$ in (11). They are exactly the two intersections of the fiber $\mathcal{F}_{(u, v)}$ with $\Sigma$. Thus, the isotropic normals define a one-to-two mapping

$$
\Omega \rightarrow \Sigma:(u, v) \mapsto\left\{\mathbf{n}_{+}(u, v), \mathbf{n}_{-}(u, v)\right\}=\mathcal{F}_{(u, v)} \cap \Sigma .
$$

The set of all isotropic normals defines the isotropic Gauss image $\Gamma=\mathbf{n}_{ \pm}(\Omega)$ of the given quadratic patch with domain $\Omega$.

If we consider the two isotropic normals along an exceptional line in the parameter domain, then we have the following two possibilities. 
- Case 1: One isotropic normal is constant along the line, while the second isotropic normal varies. The constant isotropic normal is an exceptional one with the property that several fibers $\mathcal{F}_{(u, v)}$ intersect in it.

- Case 2: Both isotropic normals are constant along the line. All points $(u, v)$ of the exceptional line possess the same fiber $\mathcal{F}_{(u, v)}$, and this fiber intersects the sphere $\Sigma$ in two exceptional isotropic normals. Consequently, the fiber is contained in $\mathcal{E}$.
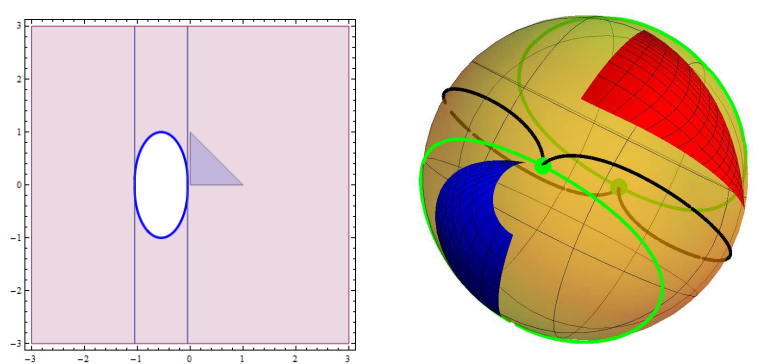

Figure 3: Left: Exceptional lines (thin blue), the curve $C(u, v)=0$ (thick blue) and the domain triangle $\triangle$. Right: The two components of the isotropic Gauss image (red and blue), the curve $\mathcal{S}_{\Sigma}$ (green), exceptional isotropic normals (green points) and the curve $\mathcal{L}_{\Sigma}$ (black).

Finally, let $\Gamma_{0} \subset \Sigma$ be the isotropic Gauss image without isotropic normals along the exceptional lines, and let $\Omega_{0}$ be the parameter domain without exceptional lines. The isotropic normals map each connected component of $\Omega_{0}$ to two connected components of $\Gamma_{0}-$ see Fig. 3. When restricted to one of the two isotropic normals, this mapping is bijective.

\subsection{Envelope computation}

We describe an algorithm for computing the exact rational envelope of a 2-parameter family of spheres given by a quadratic patch (15) over $\triangle$. Its input is a quadratic Bézier triangle (15), where all points are assumed to be space-like, i.e., $C(u, v)>0$ holds for all $(u, v) \in \triangle$. The algorithm proceeds in four steps.

Step 1: Subdivision along exceptional lines Compute the exceptional lines and triangulate the domain such that the exceptional lines are edges of the triangulation. For each triangle of the triangulation, apply a linear reparameterization of the quadratic patch such that the parameter domain is again the standard triangle $\triangle$. If the exceptional lines do not intersect the interior of the parameter domain, then no subdivision is required (see Fig. 4).

This step is similar to Algorithm 1 in [39], but the role of the parabolic lines is played by the exceptional ones. The next three steps are applied to all quadratic patches which are generated by Step 1. For these patches, at most one of the three boundary curves is an exceptional line.

Step 2: Covering the isotropic Gauss images For each of the patches obtained in Step 1, compute the isotropic Gauss images.
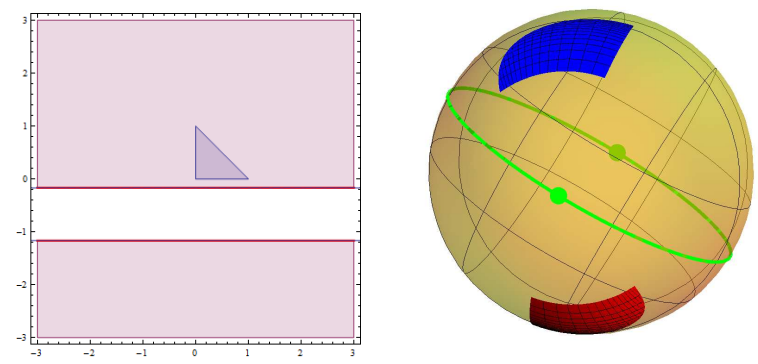

Figure 4: Left: exceptional lines (thin blue), the curve $C(u, v)=0$ (thick red) and the domain triangle $\triangle$. Right: The two components of the isotropic Gauss image (red and blue), the curve $\mathcal{S}_{\Sigma}$ (green) and exceptional isotropic normals (green points).

These Gauss images are either two spherical triangles or biangles, or a biangle and a triangle, all with curved boundaries. The biangles occur if one of the domain boundaries is an exceptional line. Next we compute rational spherical patches

$$
\mathbf{n}^{\star}: \triangle \rightarrow \Sigma:(s, t) \mapsto \mathbf{n}^{\star}(s, t)
$$

which cover the Gauss images. In the case of a biangle, one may use singular patches where the singular point coincides with the image of the exceptional line. These rational spherical patches are found using stereographic projection (see Fig. 5).

This step is similar to Algorithm 2 in [39]. As the main difference we have to consider two isotropic Gauss images instead of only one Gauss image.
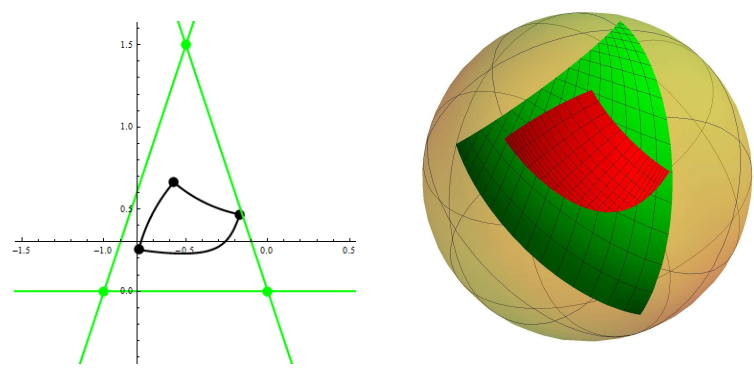

Figure 5: Left: Stereographic projection of one component of the isotropic Gauss image (black) and circumscribed triangle (green). Right: One component of the isotropic Gauss image (red) and its covering patch (green).

Step 3: Envelope computation For each spherical rational patch $\mathbf{n}^{\star}$ we compute one segment of the envelope from (7), where $\mathbf{n}$ is replaced with $\mathbf{n}^{*}$ and the parameters $(u, v)$ of the patch $\mathbf{p}$ are replaced with

$$
u(s, t)=\frac{d_{u}\left(\mathbf{n}^{\star}(s, t)\right)}{d\left(\mathbf{n}^{\star}(s, t)\right)}, \quad v(s, t)=\frac{d_{v}\left(\mathbf{n}^{\star}(s, t)\right)}{d\left(\mathbf{n}^{\star}(s, t)\right)},
$$

cf. (18) and (19). This gives rational triangular patches of degree 10. The surface patches obtained by applying the substitution (26) to (15) are MOS surfaces. 
In the case of a biangular isotropic Gauss image, the use of a spherical patch with a singular point ensures that the envelope surface possesses a regular rational parameterization, which is obtained after omitting a common factor of numerator and denominator, similar to the Example presented in Section 4.3 of [39].

Step 4: Trimming The spherical patches $\mathbf{n}^{*}$, which cover the isotropic Gauss images, are generally larger than these images, i.e., they may contain isotropic normal vectors which do not correspond to isotropic normal vectors of the MST patch over $\triangle$. Hence, we need to restrict $\triangle$ to an appropriate subset given by polynomial inequalities resulting from

$$
u(s, t) \geq 0 \wedge v(s, t) \geq 0 \wedge 1-u(s, t)-v(s, t) \geq 0,
$$

which can be derived from (26), see Fig. 6 .
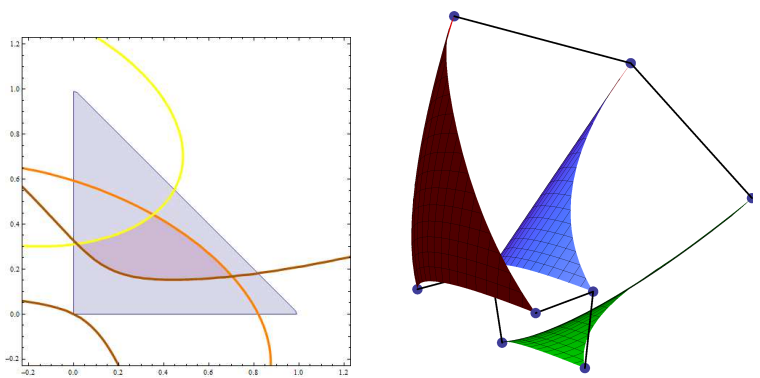

Figure 6: Left: Trimmed parameter domain of the envelope patches bounded by brown, orange and yellow curves. Right: Bisector surface (blue) and two branches of the envelope patch (red and green).

Summing up, the envelope surface consists of the trimmed patches which are generated by applying steps 2, 3 and 4 to all triangular patches obtained from Step 1 of this algorithm.

The computation of the exceptional lines leads to a polynomial equation of degree 6 , hence the solutions cannot be found in a closed form. Consequently, even if the coefficients of the input patch are rational, then the parameterizations of the envelopes which are generated by the algorithm do not possess rational coefficients.

Parameterizations with rational coefficients can be generated by using a simplified version of the algorithm, which omits the subdivision step and uses spherical patches covering the isotropic Gauss images which do not necessarily possess singular points at the exceptional isotropic normals. However, the resulting parameterizations of the envelope surfaces may possess singularities and points where the both numerators and the denominator of (26) vanish simultaneously.

We have implemented the algorithm using floating point numbers. In order to avoid numerical problems, we used the Bernstein-Bézier representations of the triangular patches. Bounds on the error introduced by an approximate computation of the exceptional lines and several examples have been presented in [60].

\section{MST approximation and trimming of offset surfaces}

We discuss the approximation of patches on the sheets of the MST of a general volume by piecewise quadratic surfaces. This approximate representation of the MST is then used for a computation of trimmed offset surfaces.

More precisely, we consider a surface patch $\mathbf{s}:[0,1]^{2} \rightarrow \mathbb{R}^{3}$ which represents a part of the boundary of the domain $V$ and which corresponds to a patch of a sheet of the MST. Consequently, for each point $\mathbf{s}(u, v)$ there is a maximal inscribed ball which touches the boundary $\delta V$ in this point and in another point.

We use the approach described in [65] for numerically computing the centers $\mathbf{c}(u, v)$ and the radii $r(u, v)$ of the maximal inscribed balls, i.e., for evaluating the points of the MST. Using this method, the surface-surface bisector problem is reduced to that of finding the common zero-set of two suitable four-variate functions in parametric uvst-space. The most important advantage of this alternative representation is that the degrees of constraint equations are considerably lower than those of the bisector surfaces in the $x y z$-space. See [65] for more details.

After generating a set of values (centers of the balls and radii), we use it to construct an approximation of the MST by a quadratic spline surface $\mathbf{P}:[0,1]^{2} \rightarrow \mathbb{R}^{3,1}$, which is defined over a criss-cross triangulation of the domain. See $[66,67,62,61]$ for more information on this type of spline functions. Alternatively, one may use quasi-interpolation methods, such as the techniques described in $[68,69,70]$. If the grid is sufficiently fine, then the piecewise quadratic approximation is guaranteed to possess only space-like tangent planes, since the first derivatives of the MST are approximated, too. In addition, the approximation order of these methods is 3 , as proved e.g. in $[67,68]$.

Next, a relation between the error in the medial surface approximation and the approximation of the associated domain boundaries can be derived as follows, cf. [47]. The Hausdorff distance between two spheres with non-negative radii $r_{1}, r_{2}$ equals $\Delta r+\Delta c$, where $\Delta r=\left|r_{1}-r_{2}\right|$ is the difference of the radii, and $\Delta c$ is the distance between the centers. Consequently, the bound on the Euclidean distance between two surfaces $\mathbf{P}, \mathbf{Q}$ in Minkowski space $\mathbb{R}^{3,1}$ implies an upper bound on the Hausdorff distance between the associated spatial domains,

$$
\mathrm{HD}\left(\Omega_{\mathbf{P}}, \Omega_{\mathbf{Q}}\right) \leq \sqrt{2} \max _{(u, v) \in[0,1]^{2}}\|\mathbf{P}(u, v)-\mathbf{Q}(u, v)\|_{E}
$$

where $\|.\| \|_{E}$ denotes the Euclidean norm. Thus, the results on the approximation order of the MST imply analogous results for the Hausdorff distance of the associated spatial domains.

Finally, combining relation (28) and the approximation order 3 of the quadratic spline approximant $\mathbf{P}$ of the MST described above yields the following result: The approximation order of the envelope algorithm presented in this paper is equal to 3 . In other words, anytime we double the number of control points in each direction, the bound on the error is reduced by the factor of 8 .

Now, we can apply the exact envelope computation method, which was described in the previous section, to each polynomial segment $\mathbf{p}$ of the quadratic spline surface $\mathbf{P}$. Recall that 

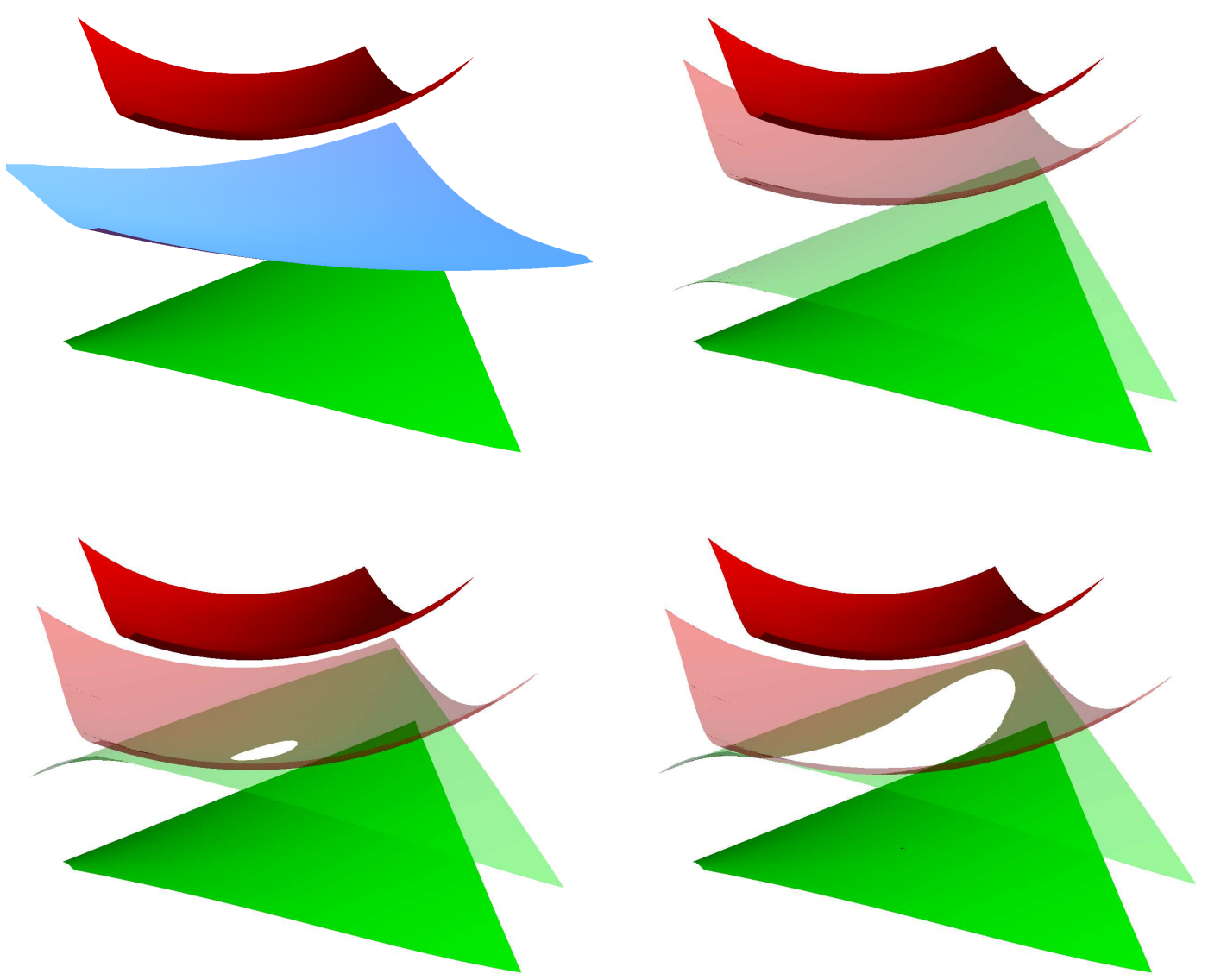

Figure 7: The bisector surface (blue) and the two branches of the envelope (red and green) in Example 2, along with the trimmed inner offsets (light red and light green) - without self-intersection (top right) and with self-intersection (bottom left and right).

the trimmed inner offsets of the boundary surface of a domain can be obtained by 1) subtracting the offsetting distance from the 4th component of the MST (which represents the radius of the maximal inscribed balls) and 2) restricting the modified MST to points with non-negative fourth components. Consequently, if the piecewise quadratic approximation $\mathbf{P}$ of the MST is available, we can use it for computing exact trimmed offsets of the boundary surface of the volume which is represented by $\mathbf{P}$. We implemented this by adding another trimming procedure to our algorithm, which restricts the piecewise quadratic MST to the domain where the radius is non-negative.

Finally, we summarize the main steps of the algorithm for computing trimmed offsets of general free-form volumes $\mathcal{V}$ with single-sheeted medial surfaces transforms.

Algorithm 1 Computation of trimmed offsets of general freeform volumes $\mathcal{V}$ with single-sheeted medial surfaces transforms.

1. Compute the centers $\mathbf{c}$ and the radii $r$ of the maximal inscribed balls of $\mathcal{V}$ for evaluating the points of the MST and choose a suitable parameterization.

2. Construct an approximation of the MST by a quadratic spline surface $\mathbf{P}$.
3. For a given distance $\delta$, compute the corresponding exact trimmed offsets of the boundary surface of the volume represented by $\mathbf{P}$ by lifting the corresponding medial surface transform in the direction of its last coordinate $r(u, v)$ and restricting the domain to points satisfying $r(u, v) \geq \delta$.

Example 2 We consider the volume $V \subset \mathbb{R}^{3}$ bounded by the two surface patches

$$
\begin{aligned}
& \mathbf{s}_{1}(u, v)=\left(u, v, \frac{1}{3} u v\right)^{\top}, \\
& \mathbf{s}_{2}(u, v)=\left(u, v, \frac{1}{3}\left(u^{2}+v^{2}\right)+1\right)^{\top},
\end{aligned}
$$

$(u, v) \in[-1,1]^{2}$, and approximate the associated MST by a piecewise quadratic surface consisting of 12 triangular patches. The maximum distance error is equal to $3.28 \%$ of the diameter of the bounding box. For each of these patches we parameterize the envelope surfaces as described in the previous section. All 12 patches of the approximation quadratic spline surface possess only space-like points and no additional subdivisions of the parameter domains are needed. Finally, Fig. 7 shows the two branches of the approximating envelope (red and green), the quadratic spline approximation of the medial surface (blue) and the two branches of inner offsets (light red and light green). 

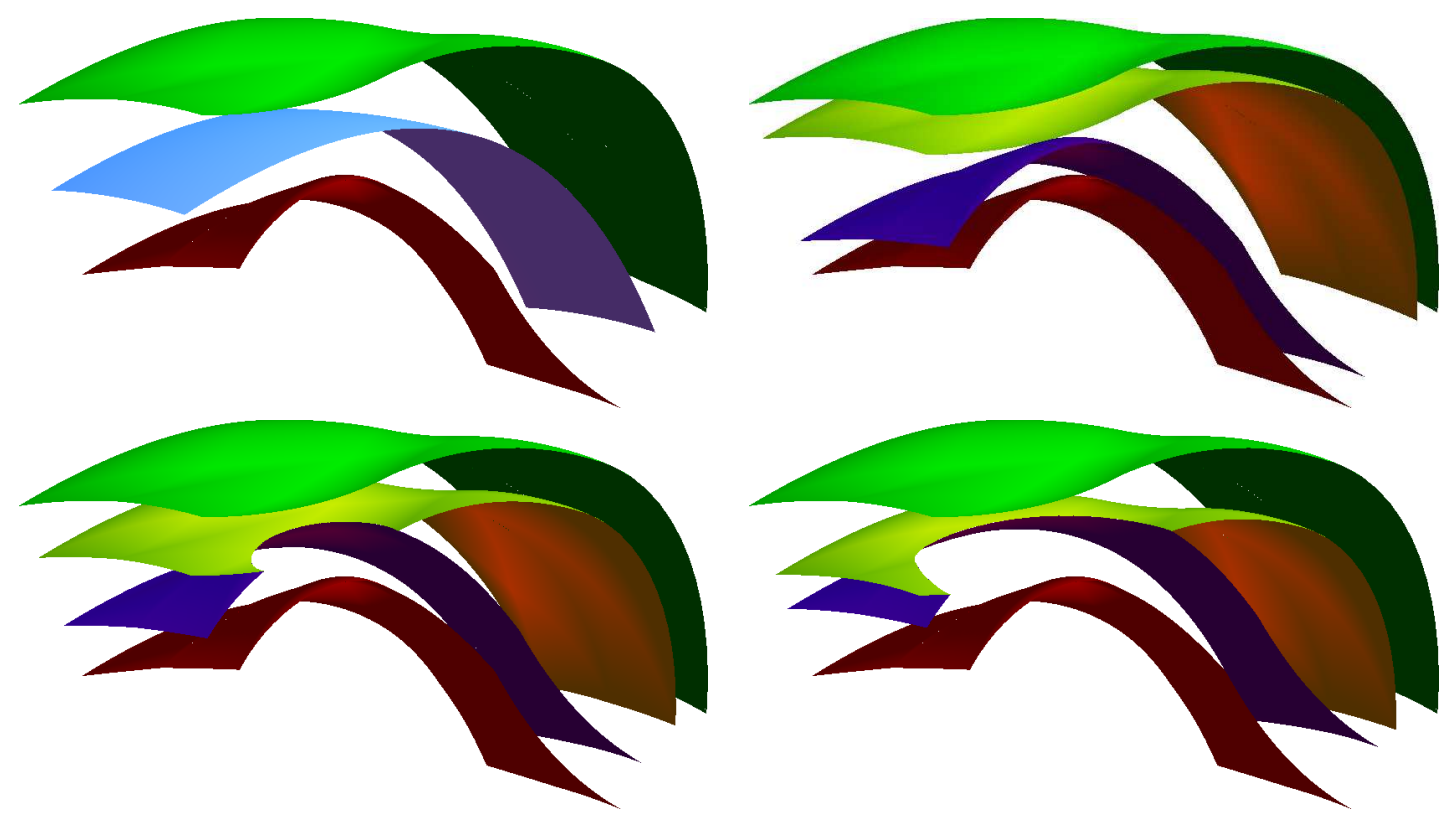

Figure 8: The bisector surface (blue) and the two branches of the envelope (red and green) in Example 3, along with the trimmed inner offsets (yellow and purple) - without self-intersection (top right) and with self-intersection (bottom left and right).

In case of the self-intersecting inner offset, the trimmed regions on the envelope are illustrated.

Example 3 We consider another volume in $\mathbb{R}^{3}$. Similarly to the previous example, we approximate its MST by a quadratic spline which consists of 24 triangular patches. The maximum distance error is equal to $1.83 \%$ of the diameter of the bounding box. Again, for each of these patches we parameterize the envelope. All patches possess only space-like points and no additional subdivision is needed. Fig. 8 shows the two branches of the approximating envelope (red and green), the quadratic spline approximation of the medial surface (blue) and two branches of inner offsets (yellow and purple) which are trimmed if the selfintersection occurs.

Next, we present two additional examples which reflect some of the difficulties of real-world situations.

Example 4 We consider a volume bounded by a watch-shaped NURBS surface of degree $(2,2)$ given by a control net of $6 \times 13$ control points and a plane (see Fig. 9 (left), the single patches of the NURBS surface are distinguished by color). The associated MST was approximated by 64 triangular patches and no additional subdivisions are needed. Fig. 9 (right) shows the two branches of the approximating envelope (red and green) and the two branches of inner offsets (yellow and purple). Inner offsets are trimmed because of their self-intersection.

Example 5 We consider a volume bounded by a NURBS surface of degree $(2,3)$ given by a control net of $4 \times 6$ control points, a plane and two canal surfaces (see Fig. 10 (left), the single patches of the NURBS surface are distinguished by color; the visible parts of the canal surfaces are displayed without parametric lines to improve contrast). The associated MST was ap- proximated by 144 triangular patches and no additional subdivisions are needed. Fig. 10 (right) presents the two branches of inner offsets (yellow and purple) of the NURBS surface which are trimmed according to their self-intersection

\section{Conclusion}

In this paper we presented an algorithm for computing boundaries and trimmed offsets of volumes given by piecewise quadratic medial surface transforms. Since polynomial quadratic patches in $\mathbb{R}^{3,1}$ belong to the class of MOS surfaces and are capable of producing $C^{1}$ approximations to free-form surfaces considered as medial surface transforms, our algorithm can be used for computing rational approximations of volume boundaries and all their offsets.

By approximating the MST surface, three main advantages are to be expected. First, the offsets of the approximation surface corresponding to the MST are represented exactly and in a globally consistent way. Second, only one approximation step is required. Once this is done, all offsets are available. Third, the trimming procedure is significantly simpler since it is achieved by imposing a polynomial inequality.

Currently, we can handle objects with an MST consisting of only one sheet. It is a challenging problem to generalize this method so that it can be applied to topologically more complex situations.

Acknowledgments B. Bastl and M. Lávička were supported by Research Plan MSM 4977751301. B. Bastl, B. Jüttler and M. Lávička were supported by grant AKTION 2009/5 (MEB060905). 

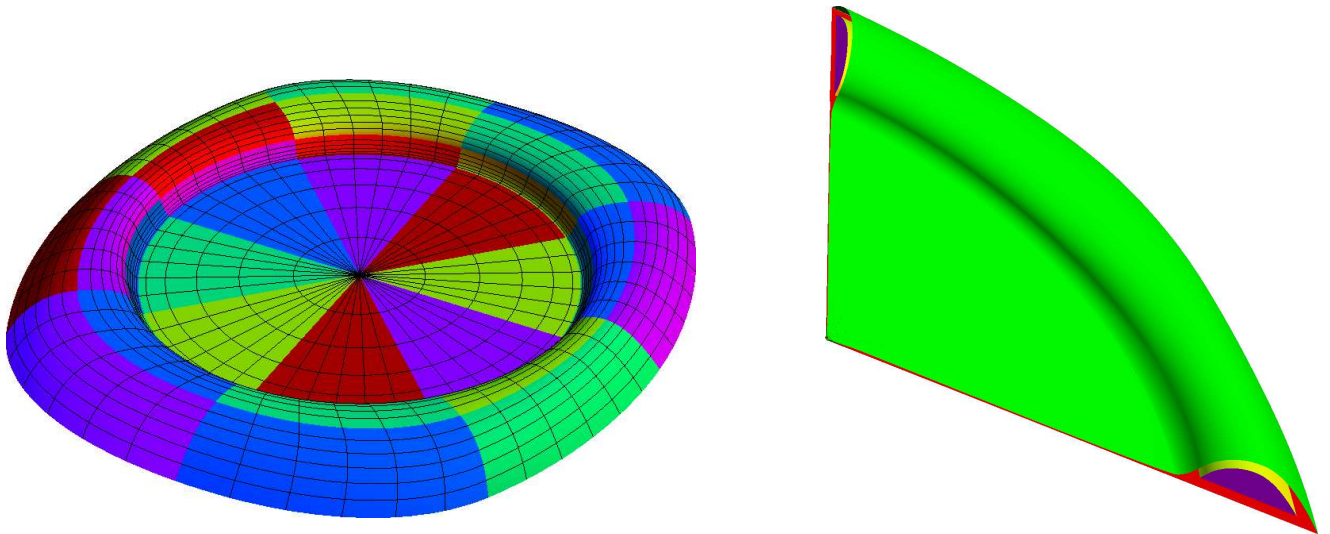

Figure 9: Left: A volume bounded by a watch-shaped NURBS surface of degree $(2,2)$ and a plane; Right: Two branches of the volume boundaries (red and green) in Example 4, along with the inner offsets (yellow and purple). Since the volume is symmetric, we present only one quarter.
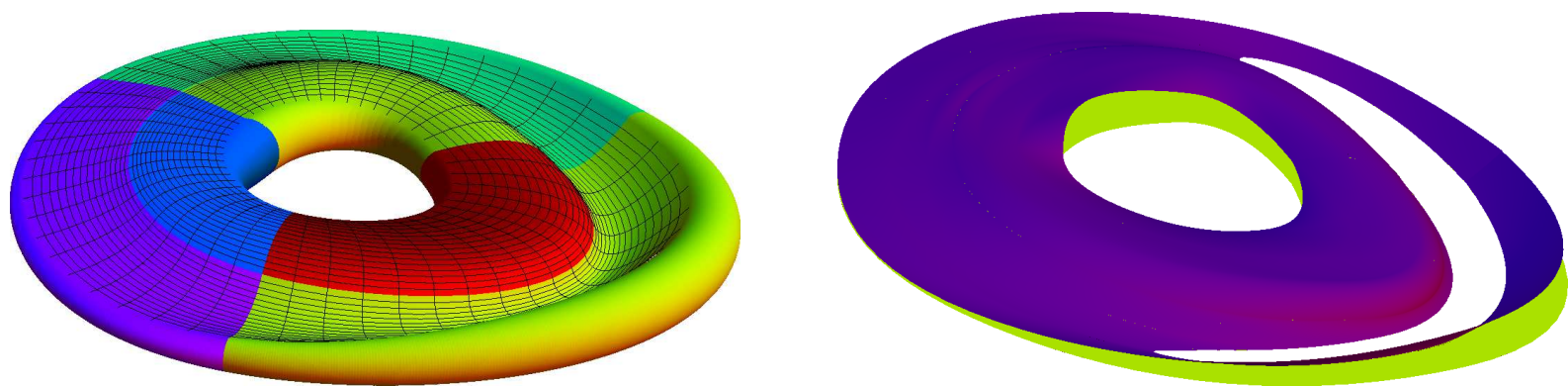

Figure 10: Left: A volume bounded by a NURBS surface of degree $(2,3)$, a plane and two canal surfaces; Right: Trimmed inner offsets (yellow and purple), see Example 5. We omitted the offsets of the canal surfaces to emphasize the offsets corresponding to the NURBS surface.

\section{References}

[1] B. Pham. Offset curves and surfaces: a brief survey. ComputerAided Design, 24:223-229, 1992.

[2] R. Kimmel and A.M. Bruckstein. Shape offsets via level sets. Computer-Aided Design, 25:154-162, 1993.

[3] G. Elber and M.-S. Kim. Special issue on offsets, sweeps, and Minkowski sums. Computer-Aided Design, 31:163, 1999.

[4] T. Maekawa. An overview of offset curves and surfaces. Computer-Aided Design, 31:165-173, 1999.

[5] L. Piegl and W. Tiller. Computing offsets of NURBS curves and surfaces. Computer-Aided Design, 31:147-156, 1999.

[6] G.V.V. Ravi Kumar, K.G. Shastry, and B.G. Prakash. Computing non-self-intersecting offsets of NURBS surfaces. Computer-Aided Design, 34:209-228, 2002.

[7] G.V.V. Ravi Kumar, K.G. Shastry, and B.G. Prakash. Computing offsets of trimmed NURBS surfaces. Computer-Aided Design, 35:411-420, 2003.

[8] G.V.V. Ravi Kumar, K.G. Shastry, and B.G. Prakash. Computing constant offsets of a NURBS B-Rep. Computer-Aided Design, 35:935-944, 2003.

[9] R.T. Farouki. The approximation of non-degenerate offset surfaces. Computer Aided Geometric Design, 3:15-43, 1986.
[10] G. Elber, I.-K. Lee, and M.-S. Kim. Comparing offset curve approximation methods. IEEE Comp. Graphics and Appl., 17:62-71, 1998.

[11] M.A. Kulczycka and L.J. Nachman. Qualitative and quantitative comparisons of B-spline offset surface approximation methods. Computer-Aided Design, 34:19-26, 2002.

[12] Z. Šŕr, R. Feichtinger, and B. Jüttler. Approximating curves and their offsets using biarcs and Pythagorean hodograph quintics. Computer-Aided Design, 38:608-618, 2006.

[13] H.-Y. Zhao and G.-J. Wang. Error analysis of reparametrization based approaches for curve offsetting. Computer-Aided Design, 39:142-148, 2007.

[14] J.-L. Shih and S.-H. Frank Chuang. One-sided offset approximation of freeform curves for interference-free NURBS machining. Computer-Aided Design, 40(9):931-937, 2008.

[15] V.F. Sun, A.Y.C Nee, and K.S. Lee. Modifying free-formed NURBS curves and surfaces for offsetting without local selfintersection. Computer-Aided Design, 36:1161-1169, 2004.

[16] J.-K. Seong, G. Elber, and M.-S. Kim. Trimming local and global self-intersections in offset curves/surfaces using distance maps. Computer-Aided Design, 38:183-193, 2006.

[17] D. Pekerman, G. Elber, and M.-S. Kim. Self-intersection detection and elimination in freeform curves and surfaces. Computer-Aided Design, 40(2):150-159, 2008. 
[18] R.T. Farouki and T. Sakkalis. Pythagorean hodographs. IBM Journal of Research and Development, (736-752), 1990.

[19] R.T. Farouki and C.A. Neff. Hermite interpolation by Pythagorean-hodograph quintics. Math. Comp., 64:1589-1609, 1995.

[20] B. Jüttler. Hermite interpolation by Pythagorean hodograph curves of degree seven. Math. Comp., 70:1089-1111, 2001.

[21] R.T. Farouki. Pythagorean-hodograph curves. In J. Hoschek, G. Farin, and M.-S. Kim, editors, Handbook of Computer Aided Geometric Design, pages 405-427. Elsevier, 2002.

[22] F. Pelosi, M.L. Sampoli, R.T. Farouki, and C. Manni. A control polygon scheme for design of planar $C^{2} \mathrm{PH}$ quintic spline curves. Computer Aided Geometric Design, 24:28-52, 2007.

[23] R.T. Farouki and T. Sakkalis. Pythagorean-hodograph space curves. Adv. Comput. Math., 2:41-66, 1994.

[24] R.T. Farouki, C. Manni, and A. Sestini. Spatial $C^{2}$ PH quintic splines. In T. Lyche, M.L. Mazure, and L.L. Schumaker, editors, Curve and Surface Design: St. Malo 2002, pages 147-156. Nashboro Press, 2003.

[25] R.T. Farouki and T. Sakkalis. Rational space curves are not "unit speed". Computer Aided Geometric Design, 24:238-240, 2007.

[26] Z. Šír and B. Jüttler. $C^{2}$ Hermite interpolation by Pythagorean hodograph space curves. Mathematics of Computation, 76:13731391, 2007.

[27] H. Pottmann. Rational curves and surfaces with rational offsets. Computer Aided Geometric Design, 12:175-192, 1998.

[28] H. Pottmann and M. Peternell. Applications of Laguerre geometry in CAGD. Computer Aided Geometric Design, 15:165-186, 1998.

[29] M. Peternell and H. Pottmann. A Laguerre geometric approach to rational offsets. Computer Aided Geometric Design, 15:223-249, 1998.

[30] W. Lü and H. Pottmann. Rational parameterization of quadrics and their offsets. Computing, 57:135-147, 1996.

[31] R. Krasauskas. Branching blend of natural quadrics based on surfaces with rational offsets. Computer Aided Geometric Design, 25:332-341, 2008

[32] M. Lávička and B. Bastl. PN surfaces and their convolutions with rational surfaces. Computer Aided Geometric Design, 25:763774, 2008.

[33] R.T. Farouki. Pythagorean-Hodograph Curves: Algebra and Geometry Inseparable. Springer, 2008.

[34] M.L. Sampoli, M. Peternell, and B. Jüttler. Rational surfaces with linear normals and their convolutions with rational surfaces. Computer Aided Geometric Design, 23:179-192, 2006.

[35] B. Jüttler. Triangular Bézier surface patches with linear normal vector field. In R. Cripps, editor, The Mathematics of Surfaces VIII, pages 431-446. Information Geometers, 1998.

[36] B. Jüttler and M.L. Sampoli. Hermite interpolation by piecewise polynomial surfaces with rational offsets. Computer Aided Geometric Design, 17:361-385, 2000.

[37] M. Lávička and B. Bastl. Rational hypersurfaces with rational convolutions. Computer Aided Geometric Design, 24:410-426, 2007.

[38] M. Peternell M and B. Odehnal. Convolution surfaces of quadratic triangular Bézier surfaces. Computer Aided Geometric Design, 25:116-129, 2008.

[39] B. Bastl, B. Jüttler, J. Kosinka, and M. Lávička. Computing exact rational offsets of quadratic triangular Bézier surface patches. Computer-Aided Design, 40:197-209, 2008.
[40] H. Blum. A transformation for extracting new descriptors of shape. In W. Wathen-Dunn, editor, Models for the perception of speech and visual form, pages 362-380. MIT Press, 1967.

[41] H.P. Moon. Minkowski Pythagorean hodographs. Computer Aided Geometric Design, 16:739-753, 1999.

[42] H.I. Choi, C.Y. Han, H.P. Moon, K.H. Roh, and N. S. Wee. Medial axis transform and offset curves by Minkowski Pythagorean hodograph curves. Computer-Aided Design, 31(59-72), 1999.

[43] H.Ch. Cho, H.I. Choi, S.-H. Kwon, D.S. Lee, and N.-S. Wee. Clifford algebra, Lorentzian geometry and rational parametrization of canal surfaces. Computer Aided Geometric Design, 21:327-339, 2004.

[44] H.I. Choi, S.W. Choi, and H.P. Moon:. Mathematical theory of medial axis transform. Pacific Journal of Mathematics, 181:5788, 1997.

[45] H.I. Choi, D.S. Lee, and H.P. Moon. Clifford algebra, spin representation and rational parameterization of curves and surfaces. Advances in Computational Mathematics, 17:5-48, 2002.

[46] G.-I. Kim and M.-H. Ahn. $C^{1}$ Hermite interpolation using MPH quartic. Computer Aided Geometric Design, 20:469-492, 2003.

[47] J. Kosinka and B. Jüttler. $G^{1}$ Hermite interpolation by Minkowski Pythagorean hodograph cubics. Computer Aided Geometric Design, 23:401-418, 2006.

[48] J. Kosinka and B. Jüttler. $C^{1}$ Hermite interpolation by Pythagorean hodograph quintics in Minkowski space. Advances in Computational Mathematics, 30:123-140, 2009.

[49] D.T. Lee. Medial axis transformation of a planar shape. IEEE Trans Pattern Analysis and Machine Intelligence, PAMI-4:362369, 1982

[50] M. Ramanathan and B. Gurumoorthy. Constructing medial axis transform of planar domains with curved boundaries. ComputerAided Design, 35:619-632, 2003.

[51] T. Culver, J. Keyser, and D. Manocha. Exact computation of the medial axis of a polyhedron. Comput. Aided Geom. Design, 21:65-98, 2004

[52] W.L.F. Degen. Exploiting curvatures to compute the medial axis for domains with smooth boundary. Computer Aided Geometric Design, 21:641-660, 2004

[53] L. Cao and J. Liu. Computation of medial axis and offset curves of curved boundaries in planar domain. Computer-Aided Design, 40:465-475, 2008.

[54] L. Cao, Z. Jia, and J. Liu:. Computation of medial axis and offset curves of curved boundaries in planar domains based on the $\mathrm{Ce}$ saro's approach. Computer Aided Geometric Design, 26(4):444454, 2009.

[55] O. Aichholzer, W. Aigner, F. Aurenhammer, T. Hackl, B. Jüttler, and M. Rabl. Medial axis computation for planar free-form shapes. Computer-Aided Design, 41(5):339-349, 2009.

[56] J. Kosinka and B. Jüttler. Mos surfaces: Medial surface transforms with rational domain boundaries. In The Mathematics of Surfaces XII, volume 4647 of Lecture Notes in Computer Science, pages 245-262. Springer, 2007.

[57] M. Peternell, B. Odehnal, and M.L. Sampoli. On quadratic twoparameter families of spheres and their envelopes. Computer Aided Geometric Design, 25:342-355, 2008.

[58] M. Peternell and B. Odehnal. On generalized LN-surfaces in 4space. In Proceedings of 'ISSAC08', pages 223-230, 2008.

[59] M. Peternell. Rational two-parameter families of spheres and rational offset surfaces. Journal of Symbolic Computation, 45(1):1$18,2010$. 
[60] B. Bastl, J. Kosinka, and M. Lávička. A symbolic-numerical envelope algorithm using quadratic mos patches. In Proceedings of the SIAM/ACM Joint Conference on Geometric and Physical Modeling, 2009.

[61] G. Nürnberger and F. Zeilfelder. Developments in bivariate spline interpolation. Journal of Computational and Applied Mathematics, 121:125-152, 2000.

[62] R.H. Wang. Multivariate spline functions and their applications. Kluwer, 2001.

[63] H. Pottmann and J. Wallner. Computational Line Geometry. Springer, 2001.

[64] J. Peters and U. Reif. The 42 equivalence classes of quadratic surfaces in affine $n$-space. Computer Aided Geometric Design, 15:459-473, 1998.

[65] G. Elber and M.-S. Kim. A computational model for nonrational bisector surfaces: Curve-surface and surface-surface bisectors. In Geometric Modeling and Processing, pages 364-372, 2000.

[66] C. de Boor, K. Höllig, and S. Riemenschneider. Box Splines. Springer, 1993.

[67] P. Sablonniéreand F. Jeeawock-Zedek. Hermite and Lagrange interpolation by quadratic splines on non-uniform criss-cross triangulations. In An international conference on curves and surfaces on Wavelets, images, and surface fitting, pages 445-452, 1994.

[68] T. Sorokina and F. Zeilfelder. Optimal quasi-interpolation by quadratic $C^{1}$-splines on type-2 triangulations. In Approximation theory XI: Gatlinburg 2004, volume 24, pages 423-438, 2005.

[69] F. Foucher and P. Sablonnière. Approximating partial derivatives of first and second order by quadratic spline quasi-interpolants on uniform meshes. Mathematics and Computers in Simulation, 77:202-208, 2008.

[70] F. Foucher and P. Sablonnière. Quadratic spline quasi-interpolants and collocation methods. Mathematics and Computers in Simulation, 79:3455-3465, 2009. 